\title{
Wireless Camera Network Demonstration for Launcher Applications Based on IR-UWB
}

\author{
André Lübken, Martin Drobczyk, Janis Sebastian Häseker, Jan Budroweit \\ Department of Avionics Systems \\ Institute of Space Systems, German Aerospace Center (DLR) \\ 28359 Bremen, Germany \\ \{andre.luebken, martin.drobczyk, janis.haeseker\}@dlr.de
}

\begin{abstract}
Sensor networks based on Impulse-Radio Ultra Wideband (IR-UWB) technology have gained traction in fields where precise localization and robust communication links are required. In spacecraft and launchers these networks can be used to connect sensors to a central on board computer or to provide a communication link between the different subsystems. This contributes to a reduced cable harness, a key driver in overall spacecraft mass and design complexity. This paper presents an application for a launcher mounted multi-camera system based on low power IRUWB sensor nodes. In combination with a modified highthroughput MAC layer from the IEEE 802.15.4 standard it is able to provide an update rate of multiple frames per second, where traditional sensor network systems would need half a minute to deliver a single frame. In addition, it is not interfering with critical telecommand/telemetry radio links of the launch vehicle due to the nature of the wideband transmission.
\end{abstract}

Index Terms-intra-spacecraft, launchers, wireless sensor networks, wireless communications, IR-UWB

\section{INTRODUCTION}

Data exchange among spacecraft subsystems is usually achieved by employing field bus systems like SpaceWire or serial point-to-point links. The necessary cable harness for these connections, however, is a significant cost driver in development and construction of these systems. In satellites, the harness contributes up to $10 \%$ to the dry mass and leads to additional launch costs [1].

Low power wireless sensor networks (WSN) can be employed to reduce the design and integration cost of these wired systems and have proven their robustness on Earth, e.g., in industrial control applications. Benefits include: (1) mitigating risks of breaking cables or connector problems, (2) easier accommodation and handling and (3) faster setup of assembly, integration and test tasks.

Recent studies have already shown wireless sensor network operation for intra-spacecraft and intra-launcher application is a viable approach ([2] and [3]). The typical setup of such a wireless sensor network (WSN) aboard a spacecraft consists of a central coordinating unit that is directly connected to the on board computer (OBC) and numerous sensor nodes distributed throughout the structure providing the data connection for the different sensors and actuators of the subsystems.

An optimal solution for such a wireless data link needs to provide a reliable, deterministic and low latency connection for sensors and actuators used in critical control systems, which was presented in [4] with the inspaWSN network stack and its medium access control (MAC) layer. For payload data acquisition with higher data rates, like the camera system presented in this paper, the MAC also provides a high throughput capability. A major problem arises from the highly reflective enclosures, in which the radio frequency (RF) components will be operated. This results in interference due to multi path fading effects when using traditional narrowband RF systems. The protocol stack used, mitigates these issues by introducing impulseradio ultra wideband (IR-UWB) in the physical layer of the stack.

In an attempt to demonstrate the capabilities of such a hybrid system, a wireless camera application for a reusable launch vehicle was developed. By using battery operated wireless camera nodes attached to the outer hull the system is extremely flexible in capturing imagery during critical flight phases from the desired positions. Challenges include the hermetic sealing of the ultra wideband transceivers as well as maintaining a stable link near the reflective surfaces of the hull in addition to interference from strong RF emitters of other subsystems in the vicinity.

The remainder of this paper is organized as follows. Section II introduces the launcher application environment and its challenges. A detailed overview of the sensor network system is given in III and the electromagnetic compatibility (EMC) considerations are presented in section IV.

\section{APPLICATION ENVIRONMENT}

Video footage from the launch phase can be an essential tool for the evaluation of flight performance and analysis of failure events. But as a component that fulfills a nonprimary function the impact on the overall vehicle design shall be minimized. This can be achieved by simplifying the interface between launcher and camera equipment. In our case this is done by mounting the camera on the outside 
of the launch vehicle. The battery powered camera node requires only a mechanical mounting point.

Because of the position on the outer surface the camera itself needs to deal with the aerothermal loads during ascent and decent, in the case of a reusable vehicle. Either the camera casing itself or an additional aerodynamic cover needs to be shaped to reduce drag. Especially the material for the view port needs to be selected according to the expected temperature range and gradient while providing high transparency in the RF and optical spectrum. The unit needs to withstand the vibration, shock and chemical environment.

Also safety concerns have a strong impact on launcher design. There is the possibility of an explosive atmosphere outside the vehicle caused by leaks or malfunction in the propulsion subsystem. Every electric unit can be a source of electric discharges or sparks that could lead to ignition. To circumvent the buildup of electric potential between different parts of the vehicle, all equipment needs to be grounded properly. Also surfaces need to have a certain conductivity to not accumulate electric charge. The electronics need to be isolated from the surrounding atmosphere either by active conditioning with gases like helium or nitrogen, or by having a hermetically sealed casing that can withstand the pressure difference that builds up during ascent. External connectors must be physically closed with caps or it must be ensured that contact surfaces are disconnected from internal circuitry and stay passive during the flight.

Finally, the overall wireless camera setup needs to deal with the strong electromagnetic environment, mainly SBand telemetry and telecommand (TMTC) links, active C-Band radar transponders and UHF/VHF links for safety systems like the flight neutralization system (FNS).

\section{SENSOR NETWORK}

\section{A. System Overview}

The network consists of a coordinator node which is located in the central avionics compartment connected to an antenna mounted in an external cable duct. The duct is RF transparent and allows a link to be established to the external camera nodes. It is also connected to the avionics system via an Ethernet connection in order for the OBC to access the collected data to downlink it.

The nodes mounted externally are installed in hermetically sealed cases with wide transparent view ports to provide a sufficient viewing angle for the cameras but also to allow the internally mounted antenna to establish a link with the coordinator.

Another critical requirement is non-interference with existing RF systems like the TMTC interface or the FNS. Hence, a sensor network based on IR-UWB was chosen for the wireless camera system.
The IR-UWB link is able to deliver live video of a few frames per second (fps) during flight over a limited capacity S-band link as well as a full 30 fps recording after landing via a WiFi ground link. The system is used not only to gather video imagery, but also to collect data from environmental sensors within the camera nodes and to assess the RF link performance during the different flight phases.

\section{B. Network Stack}

The application is based directly on a network stack comprising a modified alternative time division multiple access (TDMA) MAC layer and the IR-UWB physical (PHY) layer.

a) PHY: The foundation of the implemented network stack is built upon an IR-UWB PHY that conforms to the IEEE 802.15.4a [5] amendment of the standard which was introduced to specify alternate PHY layers of which IRUWB was chosen to provide a precision ranging capability. In addition UWB possesses a low power spectral density (PSD) avoiding interference with other RF sensitive systems. The transmission is also resilient against multi path propagation, which is a common problem for the metal enclosures of spacecraft or launchers.

The IR-UWB transceivers used for this work can be operated on several channels in the range of $3.5 \mathrm{GHz}$ to $6.5 \mathrm{GHz}$. Common narrowband technologies like $\mathrm{WiFi}$, Bluetooth or traditional 802.15.4 PHYs cause interference with other systems operating in the same frequency spectrum e.g. TMTC via S-band. IR-UWB on the other hand generates short pulses $(<2 \mathrm{~ns})$ to transmit the data. This short pulse duration thus spreads the spectrum to approx. 500-1000 MHz using the same power output, which leads to a very low PSD. Due to the low signal level for any given frequency, UWB can easily coexist with other RF applications operated in the same frequency spectrum [6]. The pulse duration also allows UWB to be nearly immune against the multi path fading effects experienced within the highly reflective metal enclosures of a spacecraft structure [7]. Compared to other available PHYs for WSNs like the classic 802.15.4 ones, WirelessHART or ISA100.11a, it provides a much higher data rate (up to $27.1 \mathrm{Mbps}$ ).

b) LLDN MAC: The robust UWB physical transmission scheme is combined with a modified version of the low latency deterministic network (LLDN) MAC layer specification proposed in the IEEE 802.15.4e [8] extension.

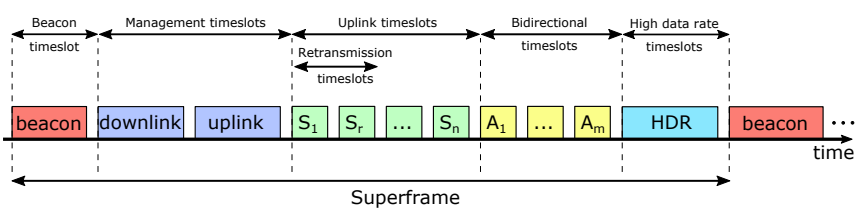

Fig. 1. The modified LLDN superframe structure 
The 802.15.4e extension proposes additional MAC layers in an attempt to add robustness to the transmission over the traditional narrowband PHYs, intended for industrial control applications. One of these MAC layers is the LLDN extension, a simple TDMA approach with fixed time slots and reduced header information. It only allows a star topology and thus deterministic and low latency for applications in control systems with strict timing requirements. The time slots in this MAC are organized in a structure called a superframe, which is marked by a beacon sent out by the network coordinator in order to synchronize the nodes and phases for management, uplink from the nodes, possible retransmissions and downlink to the nodes (Fig. 1).

A modified version of this protocol is implemented in our inspaWSN protocol stack for low power micro controllers [4]. The modifications include changes in the network management to allow the protocol to be compatible with the underlying UWB PHY. In addition our version also provides dedicated phases for high throughput (HDR) without sacrificing the low latency or deterministic behavior which allows the camera nodes to transmit high data rate video.

\section{EMC}

One of the major challenges, when using wireless systems in a space vehicle, is the electromagnetic compatibility. Spacecraft and launchers comprise very sensitive RF equipment on-board, which is used in several missioncritical applications. This becomes very important, when the on-board wireless system is also planned to be used on the outer structure of the launcher, since it can directly affect the RF signals received from the ground station or any other external source. But also the expected high power outputs of the existing RF systems on board can corrupt the communication of the onboard wireless system. For this reason, a detailed analysis of the launcher specific EMC requirements and a test campaign need to be conducted [7].

Electromagnetic compatibility design rules are not only restricted to the radiated emissions, but also include shorts for power, conducted testing, bonding and grounding [9]. However, the camera modules, which are placed on the outer structure, do not have any wired electrical interface to the launcher electronics. The radiated susceptibility and radiated emissions become the main issues to be considered for a detailed analysis.

A typical launcher includes frequencies in UHF (selfdestruct at $400 \mathrm{MHz}$ ), in L-Band (GPS) at $1.5 \mathrm{GHz}$, in S-Band (TMTC) at $2.2 \mathrm{GHz}$ and C-band (radar) at $5 \mathrm{GHz}$. One key issue of terrestrial wireless standards like $\mathrm{WiFi}$ or Bluetooth is that they operate close to the radar as well as S-Band application. IR-UWB instead uses different ultrawideband channels, where one channels center frequency is at $4 \mathrm{GHz}$ and thus does not interfere with any launcher RF systems. Nevertheless, the out-of-band emissions can lead to disturbing effects on both sides. Since the emission level of the IR-UWB signal is very low, it is well suited for the launcher environment. A test was performed in the EMC facility at the Institute of Space Systems in Bremen to show the compatibility with the general ECSS EMC requirements as well as the requirements for the Columbus module, which are comparable to the expected launcher levels. The results are shown in Fig. 2.

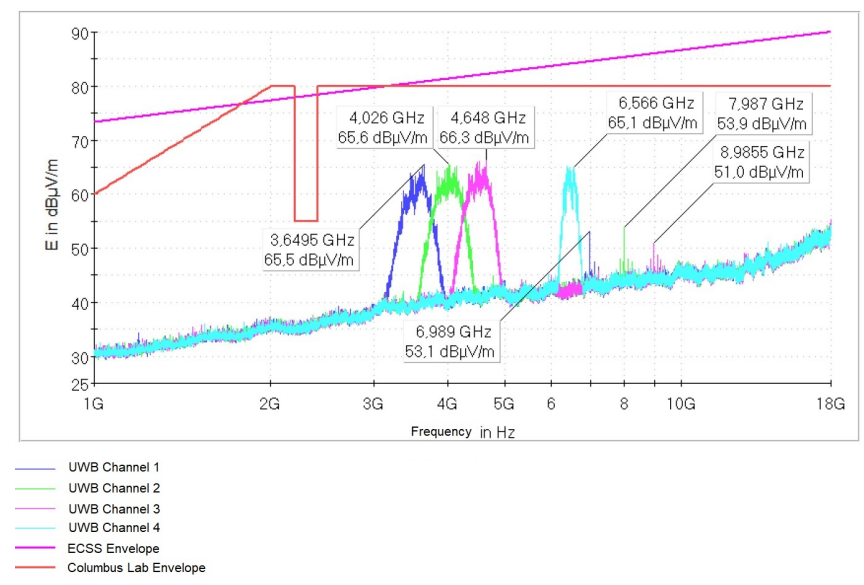

Fig. 2. Radiated emissions of the supported IR-UWB channels

It can be seen that the different channel emissions lie far below the specified limits and thus are not critical to any other relevant RF system onboard, which makes IR-UWB a well suited candidate for the proposed wireless camera network.

\section{Conclusion}

In this paper we presented a concept for a wireless camera system to be implemented for a reusable launch vehicle. Key design drivers for this system are operational safety and reliability to keep the impact on flight critical systems to a minimum.

Using robust IR-UWB with the low overhead LLDN MAC modification provides a reliable wireless link that is capable of dealing with the high data rate of the two cameras proposed for the system. The mechanical design and mounting is chosen in a way to maximize link stability while at the same time providing hermetic sealing from the harsh environment. EMC characterizations show the systems emissions rank far below the required envelope for comparable systems.

\section{REFERENCES}

[1] Rouzbeh Amini, Gerard Aalbers, Rob Hamann, Wim Jongkind, "New Generations of Spacecraft Data Handling Systems: Less Harness, more Reliability", 57th International Astronautical Congress, Valencia, Spain, 2006. 
[2] M. Drobczyk, H. Martens, "A study on low-latency wireless sensing in time-critical satellite application", presented at IEEE Sensors 2016, Orlando, USA, November 2016.

[3] H.J. Beestermöller, H.-J. Borchers, H. Luttmann, J. Sebald, M.-C. Sinnreich, M. Schneider, V. Schmid, "Wireless-Sensor Networks in Space Technology Demonstration on ISS", presented at 12 . Dresdner Sensor-Symposium 2015, Dresden, Germany, Dec 7-9 2015.

[4] Martin Drobczyk, Andre Lübken, "Novel Wireless Protocol Architecture for Intra-Spacecraft Wireless Sensor Networks (inspaWSN)", presented at 6th IEEE International Conference on Wireless for Space and Extreme Environments (WiSEE), Huntsville, USA, 2018, pp. 89-94.

[5] IEEE Std 802.15.4-2011: Part 15.4: Low-Rate Wireless Personal Area Networks (LR-WPANs), IEEE Computer Society: New York, NY, USA, 2011.

[6] M. Hirose, T. Kobayashi, A. Tomiki, T. Toda, "Effects of inner volume on UWB propagation channels within closed spaces", presented at IEEE International Conference on Ultra-WideBand (ICUWB), Paris, France, 1-3 Sept. 2014, pp.62-67.

[7] M. Drobczyk, M. Lehmann, C. Strowik, "EMC characterization of the UWB-based wireless positioning and communication experiment (wireless Compose) for the ISS", presented at IEEE WISEE 2017, Montreal, Canada, Oct 10-12, 2017.

[8] IEEE Std 802.15.4e-2012: Part 15.4: Low-Rate Wireless Personal Area Networks (LR-WPANs) (Amendment to IEEE Std 802.15.42011), IEEE Computer Society: New York, NY, USA, 2012.

[9] ECSS-E-ST-20-07C (Space Engineering - Electromagnetic Compatibility Standard), ECSS Secretariat / ESA-ESTEC, Noordwijk, The Netherlands, 2012. 